\title{
Longitudinal coupling impedance of a plane conducting ring
}

\author{
M. R. Masullo \\ INFN Sezione di Napoli, Complesso Universitario di Monte S. Angelo, I-80126, Napoli, Italy \\ G. Panariello and F. Schettino \\ Dipartimento di Ingegneria Elettronica, Università di Napoli Federico II, Via Claudio 21, I-80125, Napoli, Italy \\ V. G. Vaccaro \\ INFN Sezione di Napoli, Complesso Universitario di Monte S. Angelo, I-80126, Napoli, Italy \\ and Dipartimento di Scienze Fisiche, Università di Napoli Federico II, Complesso Universitario di Monte S. Angelo, \\ I-80126, Napoli, Italy \\ L. Verolino \\ INFN Sezione di Napoli, Complesso Universitario di Monte S. Angelo, I-80126, Napoli, Italy \\ and Dipartimento di Ingegneria Elettrica, Università di Napoli Federico II, Via Claudio 21, I-80125, Napoli, Italy \\ (Received 4 March 1999; published 30 December 1999)

\begin{abstract}
In this paper, we propose a new method to evaluate the longitudinal coupling impedance of a charged
\end{abstract} \\ particle passing perpendicularly through the center of a plane conducting ring. It is shown that the \\ solution of the problem can be expressed as a double Neumann series, whose expansion coefficients can \\ be easily computed. Moreover, we show the causality of the wake potential for a particle traveling at \\ the speed of light. \\ PACS numbers: 41.75.-i, 41.20.-q, 29.27.-a
}

\section{INTRODUCTION}

In this paper, we describe a theoretical study of the radiation emitted from a point charge $(q)$ moving at constant velocity $v=\beta c$, where $c$ is the speed of light in vacuum, and passing through the center of a perfectly conducting annular ring, with inner radius $r_{1}$ and outer radius $r_{2}$; we assume that the charge moves in the positive $z$ direction, as depicted in Fig. 1. A charge moving with uniform velocity in vacuum radiates only because of inhomogeneities existing near its path. The radiation is due to the diffraction of the field of the charge at the circular edge. The diffraction problem is described by the field traveling with the charge itself [1] and the reaction of the ring which has a traveling wave behavior. Accordingly, we can represent all the fields and/or potentials as the superposition of two terms: a term generated by the charge in the free space and a term due to the presence of the metallic region of the iris, which together have to satisfy the boundary conditions. The aim of this paper is the evaluation of the longitudinal coupling impedance due to the iris, a parameter determining the performance of an accelerator, defined by [2]

$$
Z_{\|}=-\frac{1}{q} \int_{-\infty}^{\infty} E_{z}(r=0, z ; k) e^{j k z / \beta} d z
$$

where $E_{z}(r=0, z ; k)$ represents the longitudinal component of the electric field in the frequency domain due to the reaction of the ring, and $k$ is the wave number.

Using as the unknown the Hankel transform of the radially induced surface current density on the metallic region

$$
J(r)=\int_{0}^{\infty} w F(w) J_{1}(w r) d w,
$$

the scattered electromagnetic field can be represented by the following integral transformations:

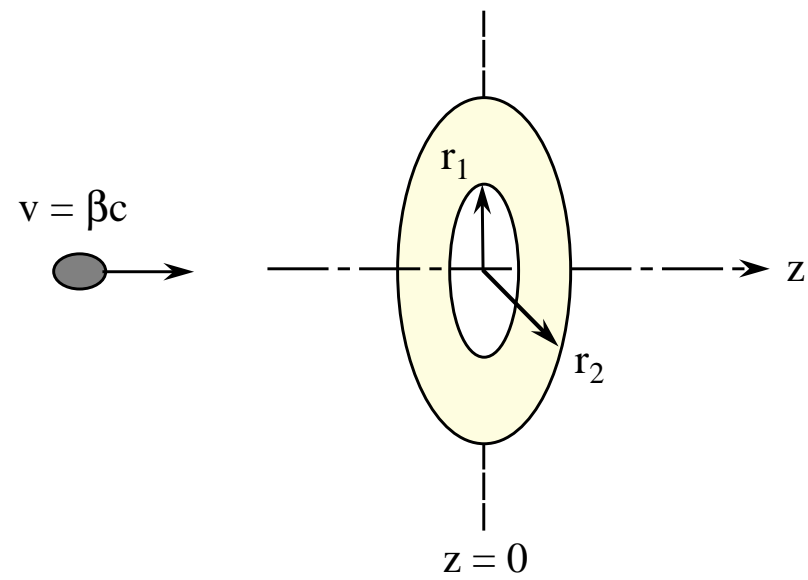

FIG. 1. (Color) The geometry of the problem. 


$$
\begin{aligned}
& H_{\varphi}(r, z)=-\frac{\operatorname{sgn}(z)}{2} \int_{0}^{\infty} w F(w) J_{1}(w r) \exp \left(-|z| \sqrt{w^{2}-k^{2}}\right) d w, \\
& E_{r}(r, z)=\frac{j \zeta_{0}}{k} \frac{\partial}{\partial z} H_{\varphi}(r, z)=\frac{j \zeta_{0}}{2 k} \int_{0}^{\infty} w \sqrt{w^{2}-k^{2}} F(w) J_{1}(w r) \exp \left(-|z| \sqrt{w^{2}-k^{2}}\right) d w, \\
& E_{z}(r, z)=-\frac{j \zeta_{0}}{k r} \frac{\partial}{\partial r}\left[r H_{\varphi}(r, z)\right]=\frac{j \zeta_{0}}{2 k} \operatorname{sgn}(z) \int_{0}^{\infty} w^{2} F(w) J_{0}(w r) \exp \left(-|z| \sqrt{w^{2}-k^{2}}\right) d w,
\end{aligned}
$$

where $\operatorname{sgn}(z)$ represents the signum function, $\zeta_{0}=120 \pi \Omega$ is the characteristic impedance of vacuum, and the branch cut is chosen such that $\operatorname{sgn}(k) \operatorname{Im}\left(\sqrt{w^{2}-k^{2}}\right) \geq 0$. By imposing the boundary conditions, the electromagnetic problem can be written as the following triple system of integral equations

$$
\begin{array}{ll}
\int_{0}^{\infty} w F(w) J_{1}(w r) d w=0, & 0 \leq r<r_{1}, \\
\int_{0}^{\infty} w F(w) \sqrt{w^{2}-k^{2}} J_{1}(w r) d w=A K_{1}(\kappa r), & r_{1}<r<r_{2}, \\
\int_{0}^{\infty} w F(w) J_{1}(w r) d w=0, & r>r_{2},
\end{array}
$$

where we called

$$
A=\frac{j q k^{2}}{\pi \beta^{2} \gamma}, \quad \kappa=\frac{k}{\beta \gamma}, \quad \gamma=\frac{1}{\sqrt{1-\beta^{2}}},
$$

$\gamma$ being the so-called Lorentz factor. The first and third equations state that there is no induced current outside the ring, while the second equation is the boundary condition for the radial component of the electric field on the metallic surface. Such a set of integral equations, all three containing the same unknown function but holding over complementary regions, is known in literature as triple integral equations. In this paper, our interest is in their application to the solution of the wave equation for diffraction problems (where the integrals are normally singular). At this point it will not be inappropriate to recall that a quite exhaustive survey of the historical developments and methods of dual equations solutions in potential theory can be found in Sneddon's book [3], but the generalization to the solution of triple problems has not yet reached satisfactory levels. We already applied [4,5] successfully some methods to the solution of dual integral problems discussed in Sneddon's book.

The longitudinal coupling impedance in terms of the unknown of the problem is given by

$$
Z_{\|}(k)=\frac{\zeta_{0}}{q \beta} \int_{0}^{\infty} F(w) \frac{w^{2}}{w^{2}+\kappa^{2}} d w
$$

The strategy of solution of triple integral equations (1.4) is similar to the strategy which was adopted for solving dual integral equations and it consists in finding a complete set of functions, each satisfying the first and third equations (1.4). In this way, the electromagnetic problem can be reduced to the only solution of the nonhomogeneous equation of the system.

\section{SOLUTION OF THE PROBLEM}

A candidate to expand the unknown spectrum $F(w)$ is the complete set $\Phi(w)=\left[\varphi_{1}(w), \varphi_{2}(w), \ldots\right]^{T}$ of functions

$$
\varphi_{n}(w)=\frac{1}{w} J_{n}\left(\frac{r_{1}+r_{2}}{2} w\right) J_{n}\left(\frac{r_{2}-r_{1}}{2} w\right)
$$

The function corresponding to $n=0$ does not satisfy the boundary condition for $r>r_{2}$ and, therefore, does not belong to this set. In fact, these functions behave [6] as previously required; namely,

$$
\int_{0}^{\infty} w \varphi_{n}(w) J_{1}(w r) d w= \begin{cases}\frac{2 \sqrt{\left(r^{2}-r_{1}^{2}\right)\left(r_{2}^{2}-r^{2}\right)}}{n \pi r\left(r_{2}^{2}-r_{1}^{2}\right)} U_{n-1}\left(\frac{r_{1}^{2}+r_{2}^{2}-2 r^{2}}{r_{2}^{2}-r_{1}^{2}}\right), & r_{1} \leq r \leq r_{2}, \\ 0, & \text { elsewhere }\end{cases}
$$

In addition, they exhibit, according to Meixner's condition, the correct edge behavior of the current; the functions $U_{n}(x)$ are the Chebyshev polynomials of the second kind. Moreover, the factor representing the edge behavior is independent from the index $n$ and can be factorized. At present, this set of functions represents the best tool to ex- pand the unknown Hankel transform of the current $F(w)$ according to the series

$$
F(w)=\frac{j q}{\pi \beta} \sum_{n=1}^{\infty} F_{n} \varphi_{n}(w)=\frac{j q}{\pi \beta} \mathbf{F}^{T} \Phi(w),
$$


which is called double Neumann series, and $\mathbf{F}=$ $\left[F_{1}, F_{2}, \ldots\right]^{T}$. Since the series (2.2) intrinsically satisfies the homogeneous equations of the system (1.4), all of the electromagnetic problem is contained in the second equation, which becomes

$$
\begin{gathered}
\frac{j q}{\pi \beta} \sum_{n=1}^{\infty} F_{n} \int_{0}^{\infty} w \sqrt{w^{2}-k^{2}} \varphi_{n}(w) J_{1}(w r) d w \\
=A K_{1}(\kappa r) .
\end{gathered}
$$

This integral equation may be solved according to the method of Rietz-Galerkin: we project it on a complete set of functions, in the domain $\left[r_{1}, r_{2}\right]$, getting an infinite system of linear algebraic equations. As the basis functions of $r$ we use just the functions on the right-hand side of Eq. (2.1). The projection consists of an inverse Hankel transform. Resorting to the integral representation of the modified Bessel function

$$
K_{1}(\kappa r)=\frac{1}{\kappa} \int_{0}^{\infty} J_{1}(w r) \frac{w^{2}}{w^{2}+\kappa^{2}} d w,
$$

and to the properties of the inverse Hankel transform of the basis functions, we get the following system of linear algebraic equations:

$$
\mathbf{A F}=\mathbf{S},
$$

where the generic element of the coefficient matrix $\mathbf{A}$ and the free term vector $\mathbf{S}$ have been defined as

$$
\begin{aligned}
A_{n m} & =A_{m n}=\frac{1}{k} \int_{0}^{\infty} w \sqrt{w^{2}-k^{2}} \varphi_{n}(w) \varphi_{m}(w) d w, \\
S_{m} & =I_{m}\left(\frac{1-a}{2} \kappa r_{2}\right) K_{m}\left(\frac{1+a}{2} \kappa r_{2}\right) .
\end{aligned}
$$

where $I_{m}(\cdot)$ and $K_{m}(\cdot)$ are the modified Bessel functions of order $m$. We note that the aspect ratio $a=r_{1} / r<1$ has been introduced and that the relevant integral,

$$
\int_{0}^{\infty} w \frac{J_{n}(x w) J_{n}(y w)}{w^{2}+\kappa^{2}} d w=I_{n}(\kappa x) K_{n}(\kappa y) \quad(x<y),
$$

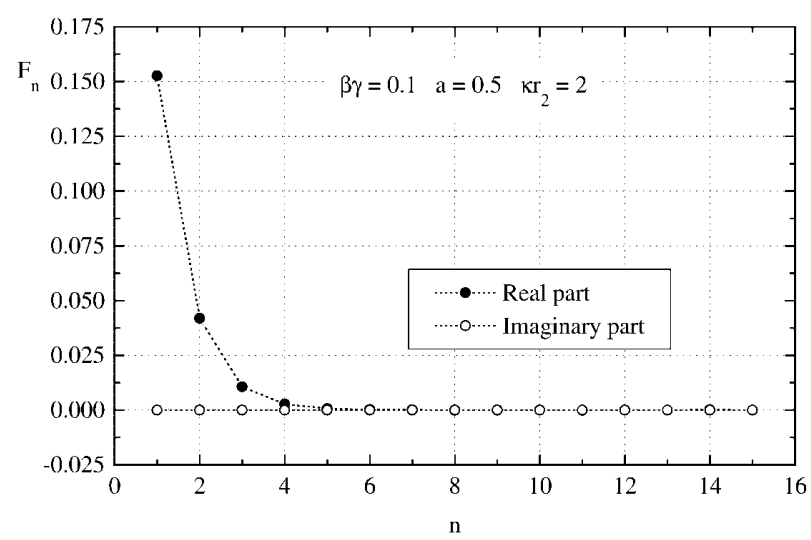

FIG. 2. Expansion coefficients as a function of the matrix dimensions for a small value of the particle speed.

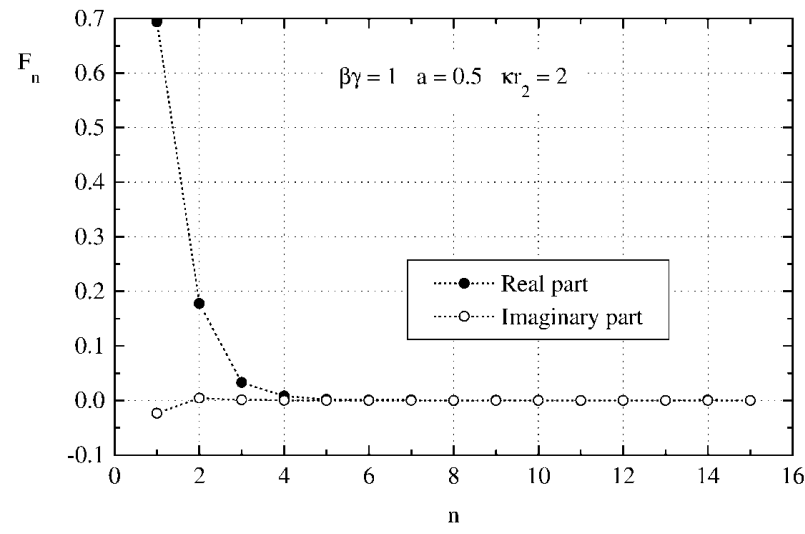

FIG. 3. Expansion coefficients as a function of the matrix dimensions for an intermediate value of the particle speed.

has been used to evaluate the free term of the system (2.5). The $A_{n m}$ were computed numerically, adopting an adaptive Gaussian quadrature routine.

As a preliminary conclusion, we can say that the problem of a particle passing perpendicularly through an annular ring has been transformed into a system of algebraic equations, whose coefficient matrix is symmetric.

The system (2.5) has been successfully adopted to evaluate the expansion coefficients in a wide range of frequencies and for various values of the particle energy. Examples are reported in Figs. 2-4 for different values of the bunch velocity, from the low value $\beta \gamma=0.1$ up to the relativistic value $\beta \gamma=10$; accordingly, the wave numbers are varied from $k r_{2}=0.2$ to 20 . These figures clearly indicate that matrices of only small size have to be inverted to evaluate the expansion coefficients and that a small number of them gives an accurate evaluation of all the relevant electromagnetic quantities, such as the current distribution density. This can be easily obtained by means of the Hankel transform (1.2) and it is represented by the

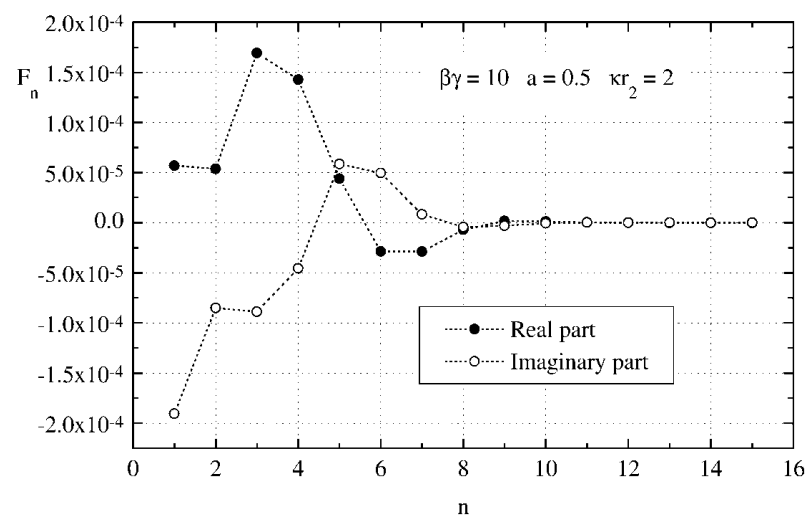

FIG. 4. Expansion coefficients as a function of the matrix dimensions for a relativistic particle. 

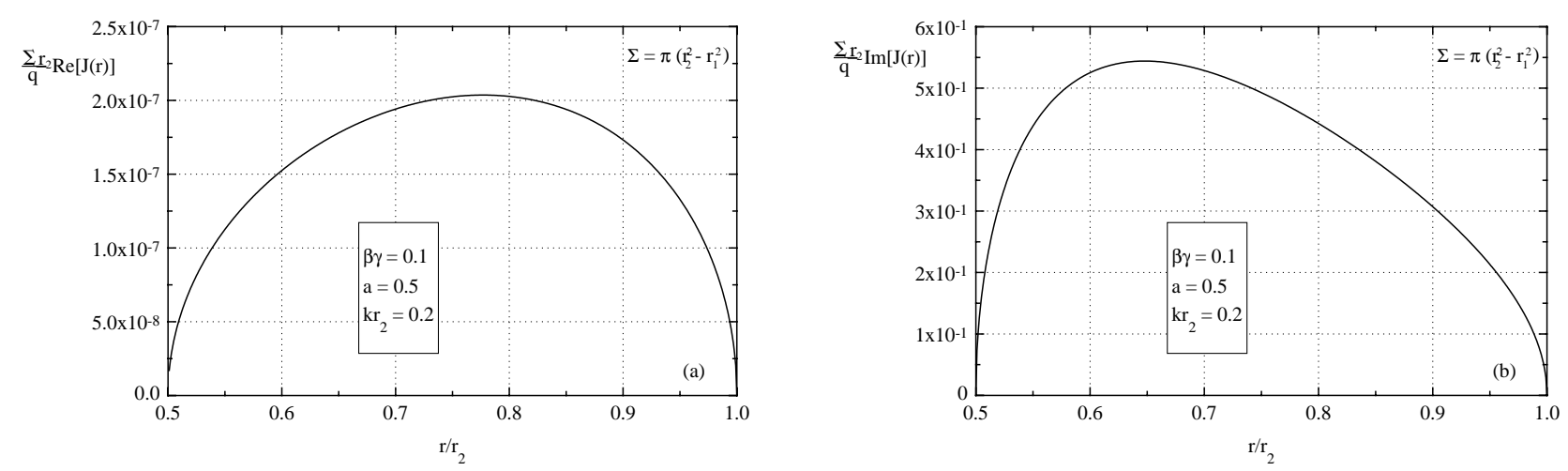

FIG. 5. An example of the current distribution density: (a) real part and (b) imaginary part.

following expansion:

$$
\begin{aligned}
J(r)= & \frac{2 j q}{\pi^{2} \beta r} \frac{\sqrt{\left(r^{2}-r_{1}^{2}\right)\left(r_{2}^{2}-r^{2}\right)}}{r_{2}^{2}-r_{1}^{2}} \\
& \times \sum_{n=1}^{\infty} \frac{F_{n}}{n} U_{n-1}\left(1-2 \frac{r^{2}-r_{1}^{2}}{r_{2}^{2}-r_{1}^{2}}\right) .
\end{aligned}
$$

An example of the current density distribution is shown in Fig. 5. It is worth noting that the current density distribution (2.8) exhibits in its first factor the correct edge behavior required by Meixner's condition.

\section{THE LONGITUDINAL COUPLING IMPEDANCE}

The longitudinal coupling impedance of an annular ring can be easily evaluated by substituting the expansion (2.2) into the definition (1.6). Using again the relevant integral (2.7), it is not difficult to conclude that the impedance is given by the following expansion:

$$
\begin{aligned}
Z_{\|}(k) & =R_{\|}(k)+j X_{\|}(k) \\
& =\frac{j \zeta_{0}}{\pi \beta^{2}} \sum_{n=1}^{\infty} F_{n} I_{n}\left(\frac{1-a}{2} \kappa r_{2}\right) K_{n}\left(\frac{1+a}{2} \kappa r_{2}\right) \\
& =\frac{j \zeta_{0}}{\pi \beta^{2}} \mathbf{S}^{T} \mathbf{A}^{-1} \mathbf{S} .
\end{aligned}
$$

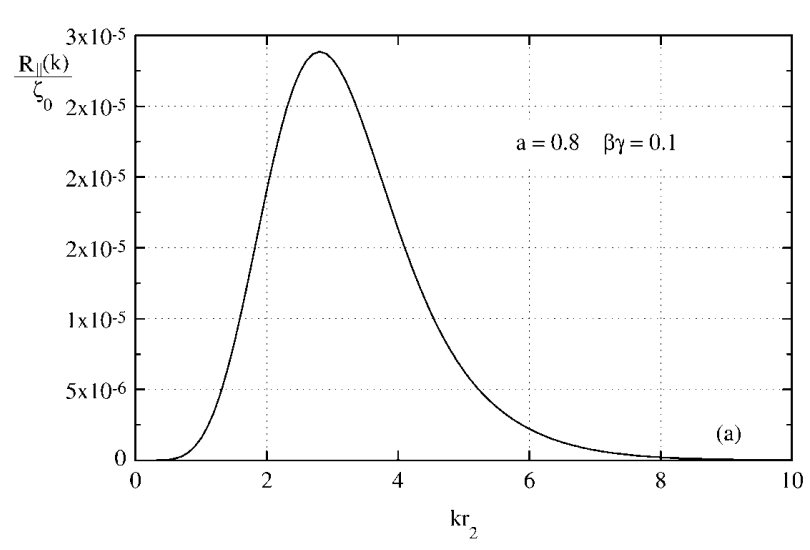

For a given value of $\gamma$, there always exists a frequency above which $\mathbf{S}$ decays exponentially with frequency, independently from $n$, provided $2 n<\kappa\left(r_{2}-r_{1}\right)$.

Figures 6 and 7 represent the longitudinal coupling impedance for a slow and a relativistic traveling particle, respectively.

\section{THE PARTICULAR CASE $\beta=1$}

Some remarks are necessary in the case $\beta=1$, namely, when the particle travels at the speed of light, because it is the particular case in most of the accelerator projects and for the slow decay of the impedance (Figs. 8 and 9). In this case, taking the limit

$$
\lim _{x \rightarrow 0} I_{n}\left(\frac{1-a}{2} x\right) K_{n}\left(\frac{1+a}{2} x\right)=\frac{1}{2 n}\left(\frac{1-a}{1+a}\right)^{n}
$$

the expansion (3.1) simplifies as

$$
Z_{\|}(k)=\frac{j \zeta_{0}}{2 \pi} \sum_{n=1}^{\infty} \frac{F_{n}}{n}\left(\frac{1-a}{1+a}\right)^{n} .
$$

In such a case, the asymptotic behavior of $\mathbf{S}$ with frequency is not the same as the previous one, so that it is

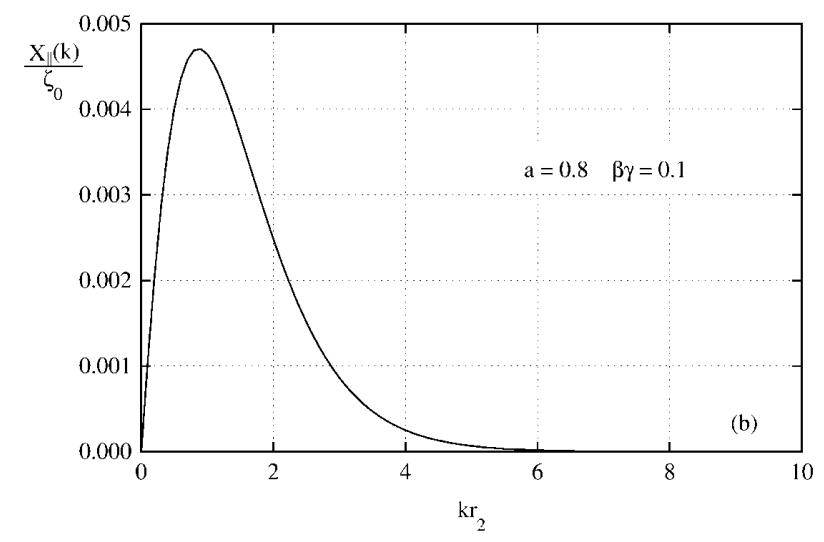

FIG. 6. Normalized values of the longitudinal coupling impedance for a slow particle: (a) real part and (b) imaginary part. 

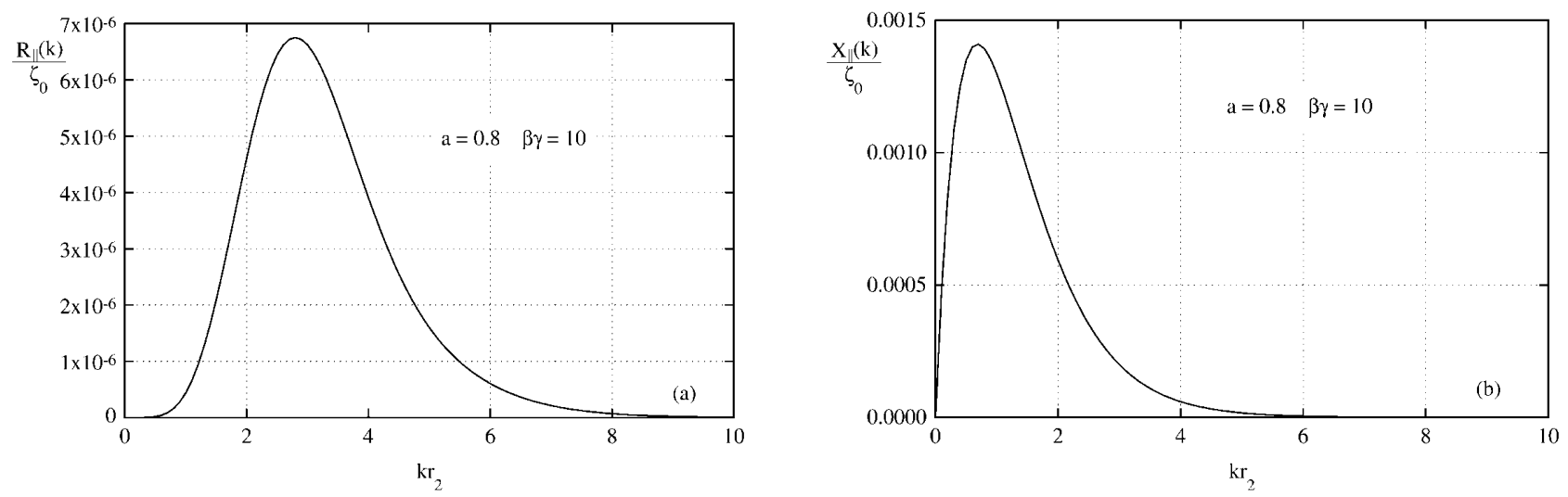

FIG. 7. Normalized values of the longitudinal coupling impedance for a relativistic particle: (a) real part and (b) imaginary part.

convenient to evaluate the asymptotic behavior $(k \rightarrow \infty)$ of the impedance.

First, we can reduce the system (1.4) to a unique integral equation. In fact, because of the following limit,

$$
\lim _{\kappa \rightarrow 0} A K_{1}(\kappa r)=\frac{j q k}{\pi r}
$$

the system can be rewritten as

$$
\int_{0}^{\infty} w F(w) J_{1}(w r) d w= \begin{cases}0, & 0 \leq r<r_{1}, \\ \int_{0}^{\infty} w F(w) \frac{j k-\sqrt{w^{2}-k^{2}}}{j k} J_{1}(w r) d w+\frac{q}{\pi r}, & r_{1}<r<r_{2}, \\ & r>r_{2} .\end{cases}
$$

Taking the inverse Hankel transform of the left-hand side of (4.4), we can easily state that the unknown $F(w)$ satisfies the integral equation

$$
F(w)=\frac{q}{\pi w}\left[J_{0}\left(w r_{1}\right)-J_{0}\left(w r_{2}\right)\right]+\int_{0}^{\infty} u F(u) \frac{j k-\sqrt{u^{2}-k^{2}}}{j k} L(w, u) d u,
$$

where the function $L(w, u)$ is defined as

$$
\begin{aligned}
L(w, u) & =\int_{r_{1}}^{r_{2}} r J_{1}(w r) J_{1}(u r) d r \\
& =\frac{u\left[r_{2} J_{1}\left(w r_{2}\right) J_{0}\left(w r_{2}\right)-r_{1} J_{1}\left(w r_{1}\right) J_{0}\left(u r_{1}\right)\right]-w\left[r_{2} J_{1}\left(u r_{2}\right) J_{0}\left(w r_{2}\right)-r_{1} J_{1}\left(u r_{1}\right) J_{0}\left(w r_{1}\right)\right]}{w^{2}-u^{2}} .
\end{aligned}
$$

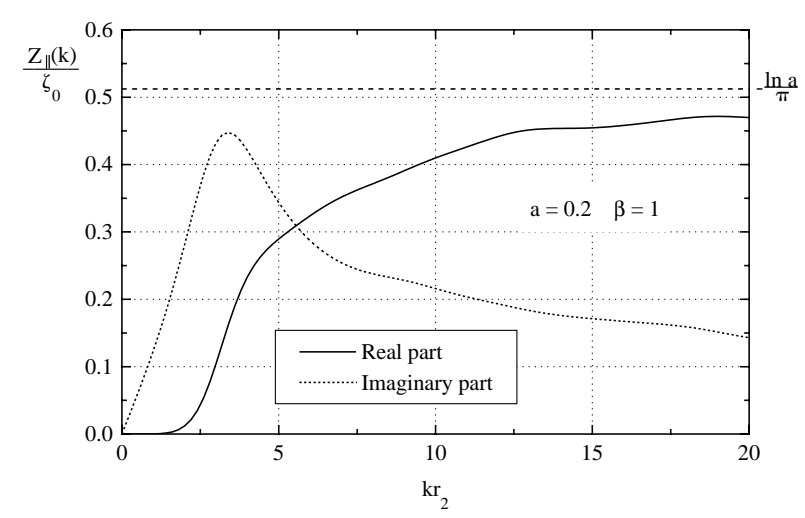

FIG. 8. Normalized values of the longitudinal coupling impedance for a particle traveling at the speed of light.
A high frequency approximation of Eq. (4.5) can be obtained in the limit of $k$ going to infinity,

$$
F(w) \rightarrow \frac{q}{\pi w}\left[J_{0}\left(w r_{1}\right)-J_{0}\left(w r_{2}\right)\right], \quad \text { when } k \rightarrow \infty .
$$

As a consequence, the longitudinal impedance (1.6) becomes

$$
\begin{aligned}
Z_{\|}(k) \rightarrow \frac{\zeta_{0}}{q} \int_{0}^{\infty} F(w) d w=\frac{\zeta_{0}}{\pi} \ln \left(\frac{r_{2}}{r_{1}}\right) & =-\frac{\zeta_{0}}{\pi} \ln a, \\
\text { when } k & \rightarrow \infty
\end{aligned}
$$

At this point we can state that we are now able to evaluate the impedance in a wide range of frequencies; this fact allows us to get an accurate estimate of the wake potential, defined as the inverse Fourier transform of the impedance 

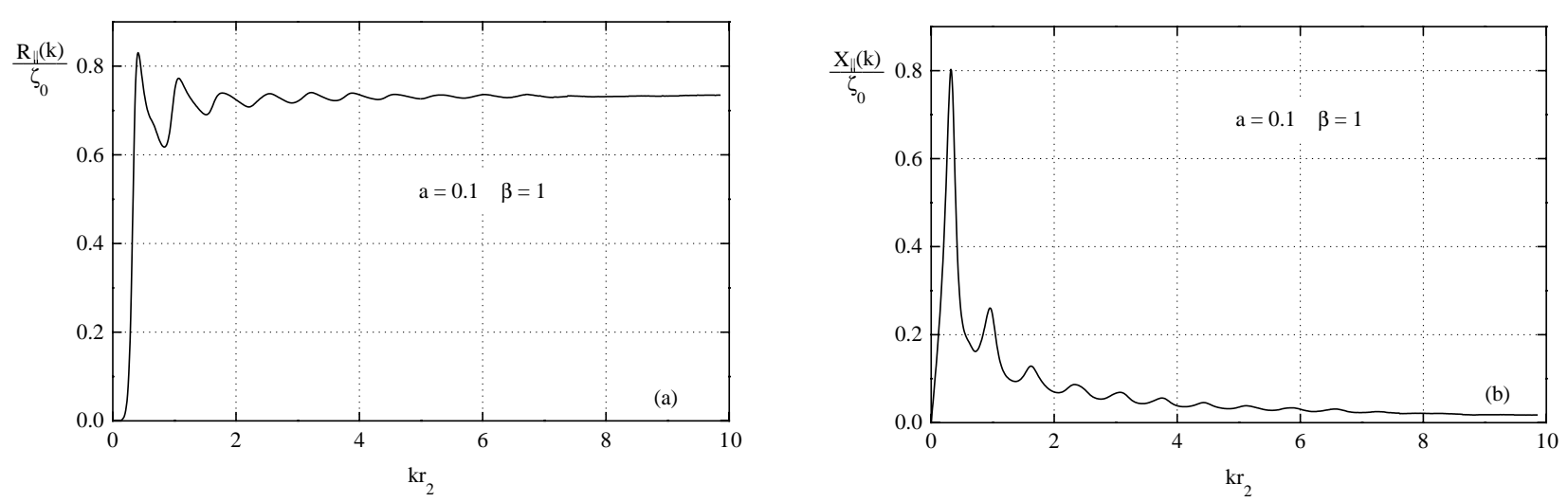

FIG. 9. Normalized values of the longitudinal coupling impedance in the case $a=0.1$ and $\beta=1$ : (a) real part and (b) imaginary part.
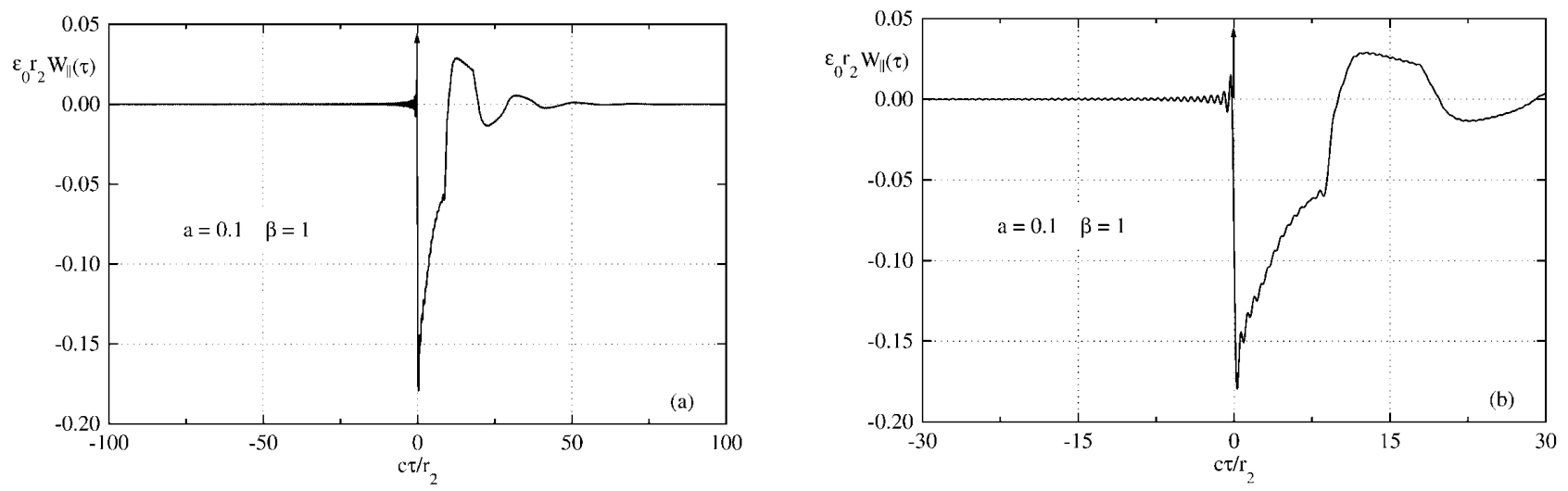

FIG. 10. Normalized values of the wake potential: (a) in the range $[-100,100]$ and (b) in the range $[-30,30]$.

$$
\varepsilon_{0} W_{\|}(\tau)=\frac{1}{2 \pi \zeta_{0}} \int_{-\infty}^{+\infty} Z_{\|}(k) \exp (j k c \tau) d k=\frac{1}{\pi \zeta_{0}} \int_{0}^{\infty}\left[R_{\|}(k) \cos (k c \tau)-X_{\|}(k) \sin (k c \tau)\right] d k
$$

In a previous paper [7], we evaluated the wake of a particle traveling through the center of a round aperture in a perfectly conducting plane; in such a case, the wake was not a causal function when $\beta=1$, but the reason of this paradox was not understood. Now we can state that the cause was the infiniteness of the plane.

Figure 10 clearly shows that the function (4.9) is a causal function, vanishing for $\tau<0$, in the case of a particle traveling at the speed of light (the arrow indicates the presence of a pulse function); in other words, the wake field is different from zero only when the electromagnetic field interacts with the iris, interaction starting at $\tau=0$.

\section{CONCLUSIONS AND PERSPECTIVES}

We presented a simple and refined way to evaluate the longitudinal coupling impedance of a perfectly conducting plane ring. The relevant electromagnetic quantities have been expanded into a double Neumann series, and, therefore, a triple boundary value problem has been transformed into a system of linear equations, able to give an accurate solution with matrices of small dimensions. We have also shown that the wake potential, namely the inverse transform of the impedance, is a causal function if the particle travels at the speed of light.

[1] G. Miano and L. Verolino, Nuovo Cimento Soc. Ital. Fis. 110B, 441-454 (1995).

[2] L. Palumbo, V. G. Vaccaro, and M. Zobov, LNF Report No. LNF-94041 (P), 1994.

[3] I. N. Sneddon, in Mixed Boundary Value Problems in Potential Theory (North-Holland, Amsterdam, 1966).

[4] G. Miano, G. Panariello, V. G. Vaccaro, and L. Verolino, COMPEL 15, 73-85 (1996).

[5] G. Miano, G. Panariello, and L. Verolino, Nuovo Cimento Soc. Ital. Fis. 113B, 243-254 (1998).

[6] I. S. Gradshtein and I. M. Ryzhik, in Table of Integrals, Series, and Products (Academic Press, New York, 1980).

[7] G. Dôme, E. Gianfelice, L. Palumbo, V. G. Vaccaro, and L. Verolino, Nuovo Cimento Soc. Ital. Fis. 104A, 12411254 (1991). 